\title{
Nonlinear stability analysis of plane Poiseuille flow by normal forms
}

\author{
A. Rauh, T. Zachrau, and J. Zoller \\ Fachbereich Physik, Carl von Ossietzky Universität Oldenburg, \\ D-26111 Oldenburg, Germany
}

October 29, 2018

\begin{abstract}
In the subcritical interval of the Reynolds number $4320 \leq R \leq$ $R_{c} \equiv 5772$, the Navier-Stokes equations of the two-dimensional plane Poiseuille flow are approximated by a 22-dimensional Galerkin representation formed from eigenfunctions of the Orr-Sommerfeld equation. The resulting dynamical system is brought into a generalized normal form which is characterized by a disposable parameter controlling the magnitude of denominators of the normal form transformation. As rigorously proved, the generalized normal form decouples into a low-dimensional dominant and a slaved subsystem. From the dominant system the critical amplitude is calculated as a function of the Reynolds number. As compared with the Landau method, which works down to $R=5300$, the phase velocity of the critical mode agrees within $1 \%$; the critical amplitude is reproduced similarly well except close to the critical point, where the maximal error is about $16 \%$. We also examine boundary conditions which partly differ from the usual ones.
\end{abstract}

\section{Introduction}

In local bifurcation theory the center manifold [1], Landau [2, 3], and normal form [4, 5, 6] methods have been invaluable tools. As a common feature, a dynamical system is approximated by series expansions around the fixed point in phase space, and in the case of the first two methods also around the critical point in parameter space. In many cases rigorous theorems are available to ensure the correct behaviour of the system in a sufficiently small neighbourhood of the critical point.

In this article we present a generalized normal form scheme with the aim to explore the subcritical, nonlinear regime in a finite neighbourhood of the fixed 
point. The method is characterized by a disposable parameter $\epsilon$ which controls the magnitude of the denominators of the normal form transformation. The case $\epsilon=0$ corresponds to the most usual normal form where resonance denominators are avoided. With $\epsilon>0$ we additionally discard quasi-resonant denominators with absolute magnitude $\leq \epsilon$. While increasing $\epsilon$ enlarges the definition domain of the transformation, the normal form gets more and more complicated by the occurrence of further nonlinear terms. Nevertheless, as rigorously proved under rather general conditions on $\epsilon$, the normal form decouples into a low-dimensional dominant and a slaved [7] subsystem in the sense that stability of the dominant system induces stability of the remaining degrees of freedom. The theorem proved generalizes previous work [8, 9].

We apply the method to the well studied subcritical stability analysis of the plane Poiseuille flow, see [3, 10, 11, 12, 13, 14, 15, 16]. The Navier-Stokes equations are approximated by a Galerkin representation in the basis of 22 eigenfunctions of the linearized part, i.e. the Orr-Sommerfeld equation. The Galerkin system, with diagonal linear part, is then subject to a normal form transformation with the resonance condition generalized through the interval parameter $\epsilon$. The latter is chosen so as to obtain a three-dimensional dominant system for the normal form in the Reynolds number interval $4320 \leq R \leq R_{c}$. The dominant system exhibits a Hopf-bifurcation at a critical amplitude $r_{c} \equiv$ $r_{c}(R)$. The normal form series expansion is carried out up to cubic monomials.

As compared with previous investigations of the plane Poiseuille flow 11, [14, 16] we examine the effect of slightly different boundary conditions: for the mode with zero wave number $q$ in downstream direction we adopt the same boundary conditions as for the other modes. Because the different modes are mixed by the nonlinear interaction, we think one should stay in the same function space for consistency.

To establish the Orr-Sommerfeld eigenfunctions of the linear problem we adopt the usual scheme of Chebyshev polynomial expansions [17]. We use 50 even and 50 odd polynomials. Eventually the critical curve $r_{c}(R)$ obtained from the normal form is transformed back into the original phase space and compared with the Landau method [12].

The definition domain of the method is principally determined by the condition that the Jacobian $J$ of the normal form transformation differs from zero in an open neighbourhood of the fixed point, where $J=1$. Because of our cut-off at cubic terms the corresponding $J$ does not allow for a reliable extrapolation. In the given case we check the definition domain in a pragmatic way by integrating the Galerkin system directly for starting points around the critical curve found by the normal form method.

We set $\alpha=\alpha_{c}=1.02$ always, which is the critical value of the basic wave number in the downstream direction at the critical Reynolds number $R=R_{c}$.

The article is organized as follows: In the following section the two-dimensional Navier-Stokes equations are expressed in terms of the scalar stream function $\Psi(x, z)$. The numerical methods to obtain the eigenfunctions of the linearized 
stationary part are briefly sketched and the new boundary conditions are stated. In section 3 the Galerkin representation is derived. In the next section our generalized normal form scheme is introduced and a decoupling theorem is formulated which is proved in Appendix B. The fifth section contains the application of the normal form scheme to the nonlinear stability analysis of the plane Poiseuille flow. In particular, the detailed normal form with numerical coefficients is given at the lowest Reynolds number considered $R=4320$. In section 6 the quantitative results are presented and compared with the results of the Landau method [12]. This is followed by a conclusion section.

\section{Basic scheme}

The two-dimensional velocity field $(u, w)$ of the incompressible plane Poiseuille flow is most conveniently described in terms of the stream function $\Psi$ as

$$
u=\frac{\partial \Psi}{\partial z} \quad ; \quad w=-\frac{\partial \Psi}{\partial x}
$$

where $u$ denotes the streamwise velocity component and $w$ the component normal to the boundaries. The Navier-Stokes equation then reads [18, 19]

$$
\frac{\partial}{\partial t} \nabla^{2} \Psi+\frac{\partial \Psi}{\partial z} \frac{\partial \nabla^{2} \Psi}{\partial x}-\frac{\partial \Psi}{\partial x} \frac{\partial \nabla^{2} \Psi}{\partial z}=\frac{1}{R} \nabla^{4} \Psi
$$

The stream function $\Psi$ is decomposed as $\Psi=\Psi^{b}+\Psi^{d}$, where the basic flow $\Psi^{b}=z-(1 / 3) z^{3}$ and the disturbance field $\Psi^{d}$ is written as a superposition of plane waves

$$
\Psi^{d}(x, z, t)=\sum_{q=-\infty}^{\infty} \Psi_{q}(z, t) \exp (i q \alpha x)
$$

with basic wave number $\alpha$. All magnitudes are dimensionless: the coordinates are measured in terms of the channel half-width $h,(u, w)$ by the maximal unperturbed velocity $U_{0}$. The Reynolds number $R=U_{0} h / \nu$ where $\nu$ denotes the kinematic viscosity. With the ansatz (3), equation (2) takes the following well-known form [3]:

$$
\begin{gathered}
L \Psi_{q}(z, t)-R \frac{\partial}{\partial t}\left(D^{2}-q^{2} \alpha^{2}\right) \Psi_{q}(z, t)= \\
-i R \alpha \sum_{q^{\prime}=-\infty}^{\infty}\left[\left(q-q^{\prime}\right) \Psi_{q-q^{\prime}}(z, t)\left(D^{3}-q^{\prime 2} \alpha^{2} D\right) \Psi_{q^{\prime}}(z, t)\right. \\
\left.-q^{\prime} D \Psi_{q-q^{\prime}}(z, t)\left(D^{2}-q^{\prime 2} \alpha^{2}\right) \Psi_{q^{\prime}}(z, t)\right]
\end{gathered}
$$

where

$$
L=\left(D^{2}-q^{2} \alpha^{2}\right)^{2}-i \alpha q R\left[2+U(z)\left(D^{2}-q^{2} \alpha^{2}\right)\right],
$$

with $D=\partial / \partial z$ and $U(z)=1-z^{2}$. 
The linearized, stationary part of (4) with $\Psi_{q}(z, t)=\Phi_{q}(z) \exp \left(\lambda_{q} t\right)$ leads to the Orr-Sommerfeld equation

$$
L \Phi_{q}(z)=\lambda_{q} R\left(D^{2}-q^{2} \alpha^{2}\right) \Phi_{q}(z) .
$$

We adopt the boundary conditions

$$
\Phi_{q}(z= \pm 1)=D \Phi_{q}(z= \pm 1)=0
$$

for all $q=0, \pm 1, \ldots$. In the case $q=0$ this is at variance with other authors [13, 14, 16] who prefer

$$
D \Phi_{q=0}(z= \pm 1)=D^{2} \Phi_{q=0}(z= \pm 1)=0 .
$$

We think the latter condition is inconsistent in the nonlinear case where it would lead to a mixing of functions with different boundary conditions. As is discussed, e.g. in [20] the boundary conditions (1) implies that the mass flux is kept constant whereas the case (8) refers to an experiment with constant pressure gradient averaged over the channel width.

For the normal form transformation to follow we need the linearized part of (田) in diagonal form, if possible. Therefore we expand the functions $\Psi_{q}$ in terms of the eigenfunctions $\Phi_{q, \nu}$ of the Orr-Sommerfeld equation, henceforth abbreviated by OSF

$$
\Psi^{d}(x, z, t)=\sum_{q, \nu} \eta_{q, \nu}(t) \Phi_{q, \nu}(z) \exp (i q \alpha x),
$$

where $\nu=1,2, \ldots$ numbers the different eigenfunctions for given wave number $q=0, \pm 1, \pm 2, \pm 3, \ldots$.

We solve the Orr-Sommerfeld equation numerically with standard procedures proposed in [17, 21] using Chebyshev polynomials as basis functions. We took 50 polynomials both for the symmetric and the antisymmetric OSF. As a check of our IMSL procedure we reproduced the eigenvalues given in [17] for the case $\alpha=1, R=10000$.

The case $q=0$ can be solved analytically. Because of our unified boundary conditions (7) we now get only antisymmetric functions, namely

$$
\Phi_{0, \nu}(z)=B_{\nu}\left[\sin \left(k_{\nu} z\right)-z \sin \left(k_{\nu}\right)\right] ; \quad \lambda_{0, \nu}=-k_{\nu}^{2} / R ; \quad k_{\nu}=\tan \left(k_{\nu}\right) .
$$

In the usual case (8) both symmetric and antisymmetric functions show up. However, one applies a kind of superselection rule suggested by numerical experience

$$
\Phi_{q, \nu}(z)=(-1)^{q+1} \Phi_{q, \nu}(-z),
$$

which excludes symmetric functions in the case $q=0$. Our antisymmetric functions differ from the traditional ones

$$
\tilde{\Phi}_{0, \nu}(z)=C_{\nu}\left[\sin (\nu \pi z)+\nu \pi z(-1)^{\nu+1}\right] ; \quad \lambda_{0, \nu}=-\nu^{2} \pi^{2} / R .
$$


Clearly, this does not affect the linear stability analysis because the critical amplitude is in the space with $q= \pm 1$. On the other hand, the different functions $\Phi_{0, \nu}$ should have an effect upon the nonlinear stability properties.

Later on we measure the strength of the disturbance field in terms of the mean energy

$$
\begin{gathered}
E(\eta)=\frac{1}{2} \overline{u^{2}+w^{2}}:=\frac{\alpha}{8 \pi} \int_{-1}^{1} d z \int_{0}^{\frac{2 \pi}{\alpha}} d x\left(u^{2}+w^{2}\right)= \\
=\frac{1}{2} \sum_{q, \nu, q^{\prime}, \nu^{\prime}} \eta_{q, \nu}(t) \eta_{q^{\prime}, \nu^{\prime}}(t) M_{q \nu, q^{\prime} \nu^{\prime}},
\end{gathered}
$$

which involves the metric components of the eigenfunctions as

$$
M_{q \nu, q^{\prime} \nu^{\prime}}=\frac{1}{2}\left[-q q^{\prime} \alpha^{2} \int_{-1}^{1} d z \Phi_{q, \nu} \Phi_{q^{\prime}, \nu^{\prime}}+\int_{-1}^{1} d z \frac{d \Phi_{q, \nu}}{d z} \frac{d \Phi_{q^{\prime}, \nu^{\prime}}}{d z}\right] \delta_{q^{\prime},-q},
$$

where $\delta$ denotes the Kronecker symbol.

\section{Galerkin representation}

In order to derive the Galerkin system for (4) we need both the right and left eigenfunctions of the Orr-Sommerfeld equation. It is convenient to introduce the abbreviations $j=(q, \nu), k=\left(q^{\prime}, \nu^{\prime}\right)$ and $l=\left(q^{\prime \prime}, \nu^{\prime \prime}\right)$, and the index projection $[j]=q,[k]=q^{\prime}$ and $[l]=q^{\prime \prime}$ and furthermore

$$
\tilde{\Phi}_{k}(z)=D \Phi_{k}(z) ; G_{k}(z)=\left(D^{2}-[k]^{2} \alpha^{2}\right) \Phi_{k}(z) ; \tilde{G}_{k}(z)=D G_{k}(z) .
$$

The Chebyshev expansion coefficients of $\Phi_{k}(z), \tilde{\Phi}_{k}(z), G_{k}(z), \tilde{G}_{k}(z)$ are denoted by $a_{n}^{k}, \tilde{a}_{n}^{k}, g_{n}^{k}, \tilde{g}_{n}^{k}$, respectively. By comparing the coefficients of the independent functions $\exp (i q \alpha x)$, we obtain

$$
\sum_{\nu}\left[\frac{d \eta_{j}}{d t}-\lambda_{j} \eta_{j}\right]\left(D^{2}-q^{2} \alpha^{2}\right) \Phi_{j}(z)=i \alpha \sum_{k, l} \eta_{k} \eta_{l}\left[\Upsilon_{k l}(z)-\tilde{\Upsilon}_{k l}(z)\right] \delta_{[k]+[l],[j]}
$$

where $\Upsilon_{k l}(z)=[k] \Phi_{k}(z) \tilde{G}_{l}(z)$ and $\tilde{\Upsilon}_{k l}(z)=[l] \tilde{\Phi}_{k}(z) G_{l}(z)$. The corresponding coefficients $v_{n}^{k l}$ and $\tilde{v}_{n}^{k l}$ are found according to the product rule given in [17]

$$
v_{n}^{k l}=\frac{[k]}{2 c_{n}} \sum_{n^{\prime}=-\infty}^{\infty} \bar{a}_{n-n^{\prime}}^{k} \overline{\tilde{g}}_{n^{\prime}}^{l} ; \tilde{v}_{n}^{k l}=\frac{[l]}{2 c_{n}} \sum_{n^{\prime}=-\infty}^{\infty} \overline{\tilde{a}}_{n-n^{\prime}}^{k} \bar{g}_{n^{\prime}}^{l} ; \quad n=0,1, \ldots
$$

with $\bar{a}_{n}=c_{|n|} a_{|n|}$ and $c_{n}=1+\delta_{n, 0}$. Furthermore, the operator $\left(D^{2}-q^{2} \alpha^{2}\right)$ is represented by the square matrix $\mathcal{D}^{q}$ as

$$
\left(D^{2}-q^{2} \alpha^{2}\right) T_{n}(z)=\sum_{n^{\prime}} \mathcal{D}_{n^{\prime} n}^{q} T_{n^{\prime}}(z)
$$


where $T_{n}(z)$ denotes the Chebyshev polynomials of order $n=0,1, \ldots$ If $q \neq 0$, then $\mathcal{D}^{q}$ is nonsingular. Its elements can be inferred from formula (A5) given in [17.

Comparing the coefficients of $T_{n}(z)$ in (16) we can write

$$
\sum_{\nu}\left[\frac{d \eta_{j}}{d t}-\lambda_{j} \eta_{j}\right] \sum_{n^{\prime}} \mathcal{D}_{n n^{\prime}}^{q} a_{n^{\prime}}^{j}=i \alpha \sum_{k, l} \eta_{k} \eta_{l}\left[v_{n}^{k l}-\tilde{v}_{n}^{k l}\right] \delta_{[k]+[l],[j]} .
$$

In the case $q \neq 0$ we multiply (19) from the left by $\left(\mathcal{D}^{q}\right)^{-1}$ and then by the $1-$ row matrix $b^{j}$ formed by the Chebyshev expansion coefficients $b_{n}^{j} n=0,1, \ldots$ of the left eigenfunction $\Phi_{j}^{L}(z)$. This gives rise to the nonlinear Galerkin system we are looking for

$$
\frac{d}{d t} \eta_{j}=\lambda_{j} \eta_{j}+\sum_{k, l} N_{j \mid k l} \eta_{k} \eta_{l}
$$

with the constant coefficients

$$
\begin{gathered}
N_{j \mid k l}=i \alpha \frac{\sum_{n} b_{n}^{j} \sum_{n^{\prime}}\left(\mathcal{D}^{q}\right)_{n n^{\prime}}^{-1}\left[v_{n^{\prime}}^{k l}-\tilde{v}_{n^{\prime}}^{k l}\right]}{\sum_{n} b_{n}^{j} a_{n}^{j}} \delta_{[k]+[l],[j]} \\
q \neq 0 ; j=(q, \nu) ; k=\left(q^{\prime}, \nu^{\prime}\right) ; l=\left(q^{\prime \prime}, \nu^{\prime \prime}\right) .
\end{gathered}
$$

The denominator is obtained from the left and right eigenfunctions as delivered by the $I M S L$ routine.

In the case $q=0$ the left and the right eigenfunctions $\Phi_{0, \nu}(z)$, given through (10), are the same. We denote the corresponding expansion coefficients by $d_{n}^{j}$ with $j=(0, \nu)$. The $N_{j \mid k l}$ are now given by

$$
N_{j \mid k l}=i \alpha \frac{\sum_{n} d_{n}^{j}\left(v_{n}^{k l}-\tilde{v}_{n}^{k l}\right)}{\sum_{n} d_{n}^{j} d_{n}^{j}} \delta_{[k]+[l],[j]} ; \quad j=(0, \nu) .
$$

Of course, for all $j$ only the symmetric part $\left(N_{j \mid k l}+N_{j \mid l k}\right) / 2$ enters (20). By the reality of the Navier-Stokes equations we have the property

$$
N_{\bar{j} \mid \bar{k} \bar{l}}=N_{j \mid k l}^{*},
$$

where the star denotes complex conjugation and $\overline{(q, \nu)}=(-q, \nu)$. As an implication the amplitudes $\eta_{j}$ obey the relation

$$
\eta_{\bar{j}}=\eta_{j}^{*} .
$$

By the Galerkin form (20) we tacitly assumed that the linear problem can be fully diagonalized. A degeneracy can be avoided by slightly shifting the Reynolds number $R$. 


\section{Interval normal form}

By means of a normal form transformation $\eta \rightarrow \xi$ we now decouple the Galerkin system (20) into a low-dimensiomal dominant system and a slaved subspace. The nonlinear, near identity transformation is written in multiindex notation as

$$
\eta_{j}=\xi_{j}+\sum_{m \in M} A_{j}(m) \xi^{m} ; A_{j}(m) \in \mathbf{C} ; j=1,2, \ldots, n,
$$

where $\xi^{m}=\xi_{1}^{m_{1}} \xi_{2}^{m_{2}} \cdots \xi_{n}^{m_{n}}$ is a monomial of order $|m|=m_{1}+m_{2}+\ldots+m_{n}$ and $M$ is the set of non-negative integer vectors

$$
M:=\left\{m\left|m \in \mathbf{N}_{0}^{n} ;\right| m \mid \geq 2\right\} .
$$

The transformed version of the Galerkin system then has the general form

$$
\dot{\xi}_{j}=\lambda_{j} \xi_{j}+\sum_{m \in M} B_{j}(m) \xi^{m},
$$

which we try to simplify in the spirit of the normal form transformation as far as possible. In practical situations like our present case it is important to avoid not only zero denominators but also near resonances, otherwise the definition domain of the transformation (25) could become unacceptably small. In view of this we introduce an interval parameter $\epsilon$ and define the generalized resonant set $\mathcal{R}_{j}(\epsilon)$. It determines the set of monomials to be kept in (27) as follows

$$
\mathcal{R}_{j}(\epsilon):=\left\{m \mid d_{j}(m) \leq \epsilon, m \in M\right\} .
$$

Here $d_{j}(m)$ is the absolute magnitude of a denominator of the normal form scheme

$$
D_{j}(m):=(m, \lambda)-\lambda_{j} ;(m, \lambda)=m_{1} \lambda_{1}+m_{2} \lambda_{2}+\ldots+m_{n} \lambda_{n} ; \quad m \in M .
$$

The coefficients $A_{j}(m)$ and $B_{j}(m)$ of (25) and of the normal form (27), respectively, are connected by recurrence relations of the following structure

$$
A_{j}(m)=-\frac{1}{D_{j}(m)}\left[B_{j}(m)+P\left(A_{k}\left(m^{\prime}\right), B_{l}\left(m^{\prime \prime}\right)\right)\right]
$$

where $P$ is a polynomial which contains only coefficients $A_{k}\left(m^{\prime}\right)$ and $B_{l}\left(m^{\prime \prime}\right)$ of lower order $\left|m^{\prime}\right|,\left|m^{\prime \prime}\right|<|m|$. If $m \in \mathcal{R}_{j}(\epsilon)$ then $d_{j}(m) \equiv\left|D_{j}(m)\right| \leq \epsilon$ and one sets the square bracket in (30) equal to zero with $B_{j}(m)=-P\left(A_{k}\left(m^{\prime}\right), B_{l}\left(m^{\prime \prime}\right)\right)$. Otherwise $B_{j}(m)$ is set equal to zero. In Appendix A we state the explicit relations for $|m|=2$ and $|m|=3$; the general recurrence relations can be found in 8 .

In qualitative normal form studies, see e.g. [4, 5, 6, 22 the interval parameter $\epsilon$ is set equal to zero. With $\epsilon \neq 0$ we now get the more complicated normal form

$$
\dot{\xi}_{j}=\lambda_{j} \xi_{j}+\sum_{m \in \mathcal{R}_{j}(\epsilon)} B_{j}(m) \xi^{m}
$$


with more nonlinear monomials as compared with the pure case $\epsilon=0$.

Nevertheless, with a suitable choice of $\epsilon$ the interval normal form (31) exhibits a useful decoupling property. To reveal this, we partition the set of eigenvalues $\lambda_{j}$ into groups characterized by their real parts. To this end the left hand complex plane of eigenvalues is divided into stripes which are parallel to the imaginary axis and of different width according to some convenient choice. The first stripe contains the least damped eigenvalues, i.e. with smallest modulus of the real part of $\lambda_{j}$. The second stripe is empty. The third stripe contains the second eigenvalue group. The fourth interval is empty, and so on. For illustration see fig. 1.

\section{Here insert fig. 1}

We label the groups by the index $\sigma$ and the members of one group by the index $\tau$. As should be noted, we have now three different equivalent labellings for the OSF: i) $j=(q, \nu)$ where $q=0, \pm 1, \pm 2, \ldots$ refers to the different

streamwise wave numbers and $\nu=1,2, \ldots$ numbers the different states at a given $q$; ii) $j=1,2, \ldots$ which numbers the different OSF ordered e.g. by decreasing real parts $\Re\left(\lambda_{j}\right)$; iii) $j=(\sigma \tau)$ which is the labelling regarding decoupling, specifically

$$
\lambda_{j}=\rho_{\sigma \tau}+i \omega_{\sigma \tau} ; \sigma=1, \ldots, \hat{n} ; \tau=1, \ldots, E_{\sigma} ; j=1, \ldots, \sum_{\sigma=1}^{\hat{n}} E_{\sigma}=n .
$$

Note that the real parts $\rho_{\sigma \tau}<0$ in this study. By convention, $\tau=1$ and $\tau=E_{\sigma}$ denote the maximum and minimum of the real parts for a given group $\sigma$, respectively. The same labelling is carried over to $m_{j} \rightarrow m_{\sigma \tau}$ and $\xi_{j} \rightarrow \xi_{\sigma \tau}$. Furthermore, we define the subspaces $\Xi_{\sigma}$ which correspond to the eigenvalues contained in one group:

$$
\Xi_{\sigma}:=\left(\xi_{\sigma 1}, \xi_{\sigma 2}, \ldots, \xi_{\sigma E_{\sigma}}\right)
$$

We now define the width $W_{\sigma}$ of a group by

$$
\begin{gathered}
W_{1}=\left|\rho_{1 E_{1}}\right| \\
W_{\sigma}=\left|\rho_{\sigma E_{\sigma}}\right|-\left|\rho_{\sigma 1}\right| \text { for } \sigma \geq 2
\end{gathered}
$$

and the distance $\Delta_{\sigma}$ between consecutive stripes containing eigenvalues by

$$
\Delta_{\sigma}=\left|\rho_{\sigma+1,1}\right|-\left|\rho_{\sigma E_{\sigma}}\right|
$$

Furthermore $\Lambda_{\sigma}$ denotes an $E_{\sigma} \times E_{\sigma}$ matrix and $P_{\sigma}$ a polynomial which starts with quadratic terms or is identically zero. As it turns out, the interval normal form decouples substantially provided the distances $\Delta_{\sigma}$ between different 
groups are sufficiently large and the width $W_{\sigma}$ of a group is sufficiently small. The precise theorem reads

Decoupling theorem: If the interval parameter $\epsilon$ allows for the properties

$$
\Delta_{\sigma}>\epsilon-\left|\rho_{11}\right| \text { for } \sigma=1,2, \ldots,
$$

and

$$
W_{\sigma}<\left|\rho_{\sigma 1}\right|-\epsilon \text { for } \sigma=2,3, \ldots,
$$

then the normal form (31) has the following structure

$$
\begin{gathered}
\dot{\Xi}_{1}=\Lambda_{1} \Xi_{1}+P_{1}\left(\Xi_{1}\right) ; \\
\dot{\Xi}_{\sigma}=\Lambda_{\sigma}\left(\Xi_{1}, \ldots, \Xi_{\sigma-1}\right) \Xi_{\sigma}+P_{\sigma}\left(\Xi_{1}, \ldots, \Xi_{\sigma-1}\right) ; \quad \sigma=2, \ldots, \hat{n} .
\end{gathered}
$$

The proof of this theorem is given in Appendix B. As should be remarked, in practical application it is advantageous to consider also the imaginary parts of the eigenvalues, which leads to a smaller resonance set $\mathcal{R}_{j}(\epsilon)$ and thus simplifies the normal form, in general.

The structure of (38), (39) implies that the equations of the higher subspaces are subsequently linear with time dependent coefficients, if the solutions of the preceding subspaces are known. Moreover, if the subsystem $\Xi_{1}$ has a stable fixed point at zero, then the fixed point is globally attractive for arbitrary initial values of the higher subspaces $\Xi_{\sigma}(t=0)$ with $\sigma=2, \ldots, \hat{n}$ provided the initial values of the subspace $\Xi_{1}$ are in the basin of attraction of its fixed point. This is exemplarily proved in Appendix $\mathrm{C}$ by means of a Lyapunovtype function. The theorem is formal as long as the existence domain of the transformation (25) is not established. If all eigenvalues are damped, then a finite convergence radius of (25) and thus a finite domain with Jacobian $J \neq 0$ can be expected; in 8 this was proved for a special choice of $\epsilon \neq 0$.

In the subcritical region with negative real parts, the condition (36) can always be fulfilled, provided the interval parameter $\epsilon$ is chosen small enough as $0<\epsilon \leq\left|\rho_{11}\right|$. In this case (37) is unnecessary, because there is no condition on the mutual distances between different groups so that we can always choose $W_{\sigma}=0$. This situation was considered in [8] where the system $\Xi_{1}$ is shown to contain only quadratic nonlinearities. However, with such a choice the parameter $\epsilon$ would become arbitrarily small in the limit $R \rightarrow R_{c}$, with the implication that the definition domain of (25) may shrink to zero.

In our application we choose $\epsilon>\left|\rho_{11}\right|$. The problem is now finding a suitable partitioning into groups which is compatible with the conditions (36), (37). Moreover, it is desirable to maintain a feasible form of the dominant subsystem $\Xi_{1}$. With the given finite cut-off of 22 eigenfunctions we have no problems finding a convenient partitioning. 


\section{Application to Poiseuille flow}

We choose the interval parameter $\epsilon$ as

$$
\begin{gathered}
\epsilon=\max \left\{\left|\lambda_{q=0, \nu=1}\right|, 2\left|\Re\left(\lambda_{q=1, \nu=1}\right)\right|\right\} \\
\epsilon<\left|\Im\left(\lambda_{q=1, \nu=1}\right)\right|
\end{gathered}
$$

where $\Re$ and $\Im$ denote the real and imaginary part, respectively. This choice provides us with a three-dimensional dominant subsystem in the Reynolds number interval $4320 \leq R \leq R_{c}$. The partitioning of eigenvalues into groups and with it the structure of the normal form is the same in this $R$-interval. The situation is quantitatively shown in fig. 1 for $R=4320$. It is compatible with the conditions (36) and (37) for the distances $\Delta_{\sigma}$ and widths $W_{\sigma}$ of the stripes. As should be noticed, our chosen interval parameter $\epsilon$ increases with decreasing Reynolds number $R$ according to the $R$-dependence of the eigenvalues.

The first stripe contains one real and the two complex conjugate eigenvalues which become critical at $R=R_{c}$. For convenience we denote the three dominant variables and eigenvalues by $\left(\xi_{1}, \xi_{2}, \xi_{3}\right)$ and $\left(\lambda_{1}, \lambda_{2}, \lambda_{3}\right)$, respectively.

Because $\lambda_{1}=\lambda_{2}^{*}$ and $\lambda_{3}=\lambda_{3}^{*}$ we have the properties $\xi_{1}=\xi_{2}^{*}$ and $\xi_{3}=\xi_{3}^{*}$. In view of the normal form (31) we introduce the abbreviations

$$
\begin{gathered}
r^{2}=\xi_{1} \xi_{2} \geq 0 \\
g_{j}\left(r^{2}\right)=\sum_{n=1}^{[\hat{N}]} B_{j}\left(n \mu_{1}+n \mu_{2}+\mu_{j}\right) r^{2 n} ; \hat{N}=\frac{\epsilon}{2\left|\rho_{11}\right|} ; j=1,2,3 \\
h\left(r^{2}\right)=\sum_{n=1}^{[\tilde{N}]} B_{3}\left(n \mu_{1}+n \mu_{2}\right) r^{2 n} ; \quad \tilde{N}=\frac{\epsilon+\left|\lambda_{3}\right|}{2\left|\rho_{11}\right|} ; \\
a_{k}=2 N_{k 13} ; \quad k=1,2
\end{gathered}
$$

where $[\hat{N}]$ and $[\tilde{N}]$ denote the integer parts of $\hat{N}$ and $\tilde{N}$, respectively. With this, in the subspace $\Xi_{1}$ the normal form (31) has the following structure

$$
\begin{gathered}
\dot{\xi}_{1}=\left[\lambda_{1}+a_{1} \xi_{3}+g_{1}\left(r^{2}\right)\right] \xi_{1} \\
\dot{\xi}_{2}=\left[\lambda_{2}+a_{2} \xi_{3}+g_{2}\left(r^{2}\right)\right] \xi_{2} \\
\dot{\xi}_{3}=\lambda_{3} \xi_{3}+h\left(r^{2}\right)+g_{3}\left(r^{2}\right) \xi_{3}
\end{gathered}
$$

Obviously, the above system is decoupled from the remaining variables $\xi_{k}$ with $k \geq 4$.

In the following we take the transformation (25) and with it the normal form (31) up to cubic terms. With the aid of polar coordinates $\xi_{1}=r \exp (i \varphi)$ and by setting $\xi_{3} \equiv \xi, \lambda_{3} \equiv \lambda, \rho_{11} \equiv \rho$ and $\omega_{11} \equiv \omega$ the transformed dominant system is further reduced to effectively two dimensions

$$
\dot{r}=r\left[\rho+\Re\left(a_{1}\right) \xi+b r^{2}\right] ; r \geq 0 ;
$$




$$
\begin{gathered}
\dot{\xi}=\lambda \xi+\gamma_{1} r^{2}+\gamma_{2} r^{2} \xi ; \\
\dot{\varphi}=\omega+\beta r^{2} .
\end{gathered}
$$

As illustrated in fig. 2, we obtain a stable fixed point at $r=\xi=0$ and a further fixed point $F^{*}$ at $\left(r_{c}, \xi_{c}\right)$, which corresponds to a Hopf limit cycle 23 with angular frequency $\dot{\varphi}$ given by (48).

Near the critical point $R=R_{c}$ we have $\xi_{c}=-\gamma_{1} r_{c}^{2} / \lambda$ which renormalizes (46) to $\dot{r}=r\left[\rho+b_{L}\left(R_{c}\right) r^{2}\right]$ with the Landau-type parameter at $R=R_{c}$

$$
b_{L}=b-\frac{\gamma_{1} \Re\left(a_{1}\right)}{\lambda} .
$$

For higher order dominant systems there enter further renormalizing summands into $b_{L}$.

From table 1 it is seen, that the Landau parameter $b_{L}$ of our normal form method is almost independent of the choice of the interval parameter $\epsilon$, as it should be.

Table 1

Dependence of the Landau parameter $b_{L}$ on the choice of the interval parameter $\epsilon$ at $R=5770$ with $E_{1}$ denoting the dimension of the corresponding dominant system. The main results of the paper are for $E_{1}=3$.

\begin{tabular}{c|c|c|c}
\hline$\epsilon$ & $\left|\lambda_{q=0, \nu=2}\right|$ & $\left|\lambda_{q=0, \nu=1}\right|$ & $2\left|\Re\left(\lambda_{q=1, \nu=1}\right)\right|$ \\
$b_{L}$ & 39.163 & 39.168 & 39.174 \\
$E_{1}$ & 5 & 3 & 2 \\
\hline
\end{tabular}

As can be inferred from fig. 2, the basin of attraction of the zero fixed point is limited by the curve $\dot{r}=0$. If the starting point lies inside this basin of attraction, both the variables of the dominant and the slaved system go to zero.

\section{Here insert fig. 2}

Let us examine the slaved system at the non-zero fixed point $(r, \xi)=$ $\left(r_{c}, \xi_{c}\right)$. To this end we write down in the following the full normal form of our Galerkin system at $R=4320$ with the first two equations referring to the dominant system (46), (47)

$$
\begin{aligned}
& \dot{r}=r\left(\rho+0.02942 \xi+46.64 r^{2}\right) \quad ; r \geq 0 \\
& \dot{\xi}=\xi\left(\lambda-2.568 r^{2}\right)+0.6561 r^{2}
\end{aligned}
$$




$$
\begin{aligned}
\dot{\xi}_{4} & =\xi_{4}\left(\lambda_{4}-58.43 r^{2}\right)-75.72 \xi r^{2} \\
\dot{\xi}_{5} & =\xi_{5}\left(\lambda_{5}-110.19 r^{2}\right)-65.94 \xi_{4} r^{2} \\
\dot{\xi}_{6} & =\xi_{6}\left(\lambda_{6}+80.37 r^{2}\right) \\
\dot{r}_{1} & =r_{1}\left(\rho_{1}-0.06202 \xi+12.39 r^{2}\right) \quad ; \quad r_{1} \geq 0 \\
\dot{\xi}_{9} & =\xi_{9}\left(\lambda_{9}+388.6 r^{2}\right) \\
\dot{r}_{2} & =r_{2}\left(\rho_{2}-0.08132 \xi+83.36 r^{2}\right) \quad ; \quad r_{2} \geq 0 \\
\dot{r}_{3} & =r_{3}\left(\rho_{3}-0.09851 \xi-206.2 r^{2}\right) \quad ; \quad r_{3} \geq 0 \\
\dot{\xi}_{14} & =\xi_{14}\left(\lambda_{14}+168.2 r^{2}\right) \\
\dot{\xi}_{15} & =\xi_{15}\left(\lambda_{15}-155.95 r^{2}\right)+2561 \xi_{4} r_{1}^{2} \\
\dot{\xi}_{16} & =\xi_{16}\left(\lambda_{16}-61.77 r^{2}\right)-86210 \xi_{4} r_{2}^{2} \\
\dot{\xi}_{17} & =\xi_{17}\left(\lambda_{17}+14.79 r^{2}\right)+15619 \xi_{5} r_{3}^{2}+63.38 \xi_{14} r_{1}^{2} \\
\dot{\xi}_{18} & =\xi_{18}\left(\lambda_{18}+0.6672 r^{2}\right)+19360 \xi_{14} r_{2}^{2}+5671 \xi_{9} r_{3}^{2} \\
\dot{\xi}_{19} & =\xi_{19}\left(\lambda_{19}-1.430 r^{2}\right)+1280 \xi_{15} r_{3}^{2} \\
\dot{\xi}_{20} & =\xi_{20}\left(\lambda_{20}-1.772 r^{2}\right)-4.482 \xi_{18} r_{1}^{2}-238.51 \xi_{17} r_{2}^{2} \\
\dot{\xi}_{21} & =\xi_{21}\left(\lambda_{21}-1.095 r^{2}\right)-0.7456 \xi_{19} r_{1}^{2} \\
\dot{\xi}_{22} & =\xi_{22}\left(\lambda_{22}-0.3739 r^{2}\right)-172.1 \xi_{19} r_{3}^{2},
\end{aligned}
$$

where the magnitudes $\rho=\Re\left(\lambda_{1}\right), \lambda=\lambda_{3}, \lambda_{4}, \ldots, \lambda_{22}$ of the linear problem are listed in Appendix D. As in the case of the dominant system, polar coordinates are used for the complex conjugate amplitudes with $r_{1}, r_{2}$ and $r_{3}$ denoting the moduli of the modes $(q= \pm 1, \nu=2),(q= \pm 2, \nu=1)$ and $(q= \pm 3, \nu=1)$, respectively. The equations for the corresponding polar angles are omitted. The equations (52) and (53) have nonzero fixed points $\xi_{4}^{c}$ and $\xi_{5}^{c}$, respectively, whereas each of the remaining equations has fixed point zero. Thus the overall nonzero fixed point at $R=4320$ is given by

$$
\begin{gathered}
\left(r_{c}=0.008231, \xi_{c}=0.009170, \xi_{4}^{c}=-0.002647,\right. \\
\left.\xi_{5}^{c}=0.0003380, \xi_{6}^{c}=0, \ldots, \xi_{22}^{c}=0\right) .
\end{gathered}
$$

When the variables $r$ and $\xi$ are substituted by $r_{c}$ and $\xi_{c}$ in the slaved equations (52) -(67), then it turns out that the separate fixed point of the slaved system is stable. Therefore, at the critical point of the dominant subsystem there is no runaway of the slaved variables. We can thus define a meaningful critical energy by mapping the coordinates of the nonzero fixed point back into the original phase space of the amplitudes $\eta$. With the aid of (13) we obtain $E=0.26 * 10^{-3}$ at $R=4320$ which has to be compared with the unperturbed energy $E_{0}=8 / 15$ of the basic flow. The relative critical energy $E / E_{0}$ has the order of magnitude established in the literature, see e.g. [13].

Regarding the fixed point in the original phase space, we state the maximal amplitude for a given wave number $q$

$$
\left|\eta_{q=0}^{\max }\right|=9.176 * 10^{-3} ;\left|\eta_{q=1}^{\max }\right|=5.726 * 10^{-3}
$$




$$
\left|\eta_{q=2}^{\max }\right|=2.630 * 10^{-8} ;\left|\eta_{q=3}^{\max }\right|=3.577 * 10^{-9} .
$$

As a final remark of this section, it is straightforward to extend the given formalism to Reynolds numbers below $R=4320$ by choosing a larger interval parameter $\epsilon$. This amounts to adding further nonlinear terms to the normal form so as to preserve the same structure in the larger Reynolds number interval.

\section{Comparison with Landau method}

Our main results are represented by fig. 3 . We adopt the same normalization

$$
\Phi_{q=1, \nu=1}(z=0)=1
$$

as used in the Landau method [11, 12].

\section{Here insert fig. 3}

As is seen both the critical curve of the Landau method and of our normal form approach are consistent with the neutral curve of the Galerkin system, which is approximated by directly integrating the Galerkin system. The starting points were choosen close to the critical amplitude vector $\eta_{s}$, which is the image of the fixed point $F^{*}$ of the normal form space. This result also indicates that, in view of the Galerkin system choosen, our function space of 22 OrrSommerfeld eigenfunctions is sufficiently large. However the Galerkin space is to small to reproduce the Landau method results near the critical point for $5760 \leq R \leq R_{c}$. We find $b_{L}\left(R_{c}\right)=39.17$, which is by $36 \%$ larger than the corresponding value, 28.88, obtained by the Landau method [12]; correspondingly at the critical Reynolds number our critical amplitude is by $16 \%$ smaller, see fig. 3 and fig. 4. As should be remarked the critical curve of the Landau method [12 is established in the smaller interval $5300 \leq R \leq R_{c}$.

For comparison with the results reported elsewhere [12] we give the angular frequency $\dot{\varphi}$ of the nonlinear wave at the fixed point $F^{*}$ for several Reynolds numbers. As table 2 shows, the numerical values agree within $1 \%$.

Table 2

Comparison of the angular frequency of the nonlinear wave with the Landau method.

\begin{tabular}{c|ccccc}
\hline $\mathrm{R}$ & 5200 & 5400 & 5500 & 5700 & 5770 \\
\hline$\dot{\varphi}$ & 0.2771 & 0.2744 & 0.2730 & 0.2704 & 0.2694 \\
$\dot{\varphi}_{\text {Landau }}$ & 0.2743 & 0.2725 & 0.2717 & 0.2699 & 0.2693 \\
\hline
\end{tabular}


In fig. 4 we show results for the parameter $b_{L}$ as a function of the number $N$ of modes with $q=0$ considered. The new boundary conditions, curve (2), and the usual ones, curve (1), give practically the same value $b_{L}$.

\section{Here insert fig. 4}

As to the definition domain of our normal form transformation we cannot safely rely on the criterion of the Jacobian. In the given case the Jacobian is larger 1 well beyond the critical amplitude.

\section{Conclusions}

A generalized normal form scheme for dissipative flows was presented which allows to control the minimal magnitude of the normal form denominators, and at the same time keeps a useful decoupling property of the normal form. The decoupling property was rigorously proved and constitutes a new result. The feasibility of the method was examined for the well studied example of plane Poiseuille flow in the subcritical interval $4320 \leq R \leq R_{c}$ of the Reynolds number $R$. The critical energy $E_{c}(R)$ or equivalently the critical amplitude $\eta_{c}(R)$, at which the laminar flow becomes unstable, agrees well with the results obtained by other methods [12], except close to the critical point where our critical amplitude is about $16 \%$ smaller than the corresponding value of the Landau method [12]. The latter is established in the interval $5300 \leq R \leq R_{c}$, whereas the present normal form gives reliable results down to $R=4320,0$ see fig. 3 .

The function space, in our case the Orr-Sommerfeld eigenfunctions, is rigorously determined in principle for every parameter point $R$. For comparison, both the Landau and the center manifold method rely on a critical parameter point $R_{c}$ of the linearized problem around which one expands in powers of $\sqrt{R_{c}-R}$, for instance. The normal form method, on the other hand, requires the existence of a stable stationary solution only which provides a fixed point in phase space. Therefore the present method should be useful to study e. g. pipe flow or plane Couette flow for which the linearized system lacks a critical point.

As a minor detail we studied boundary conditions which differ from the usual ones for the modes with $q=0$. Our new boundary conditions constitute a unified function space for all modes. However it turns out that both boundary conditions give practically the same results, see fig.4.

The quantitative validation of an existence domain is a notoriously difficult problem in all three discussed methods. We found our normal form results being consistent with results from the direct integration of the Galerkin system. 
At the critical curve $r_{c}(R)$ we found the Jacobian of the normal form transformation being always positive and well away from zero. However, since our normal form transformation neglects higher than cubic terms, the Jacobian criterion is not safe here.

The numerical efforts reside to about $90 \%$ in the normal form method, whereas the remaining computing time lies mainly in establishing the OrrSommerfeld eigenfunctions and the Galerkin system. For one parameter value $R$ the computing time was about six minutes on a RISC 6000 work station.

The parameter interval $4320 \leq R \leq R_{c}$ considered was determined by the choice of the interval parameter $\epsilon$ together with the requirement that the normal form (31) keeps the same structure in the whole parameter interval. It is in principle possible to go deeper into the subcritical range by choosing a larger $\epsilon$ which would result in a higher dimensional dominant subsystem.

\section{Appendix A: Recurrence relations}

We insert the normal form transformation (25) into the Galerkin system (20) and consider the normalized dynamical system (27) as an ansatz. The left hand side of (20) then reads

$$
\begin{aligned}
& \dot{\eta}_{j}=\lambda_{j} \xi_{j}+\sum_{m \in M}\left[(m, \lambda) A_{j}(m)+B_{j}(m)\right] \xi^{m} \\
& +\sum_{m, m^{\prime} \in M} \sum_{l} A_{j}\left(m^{\prime}\right) m_{l}^{\prime} B_{l}\left(m-m^{\prime}+\mu_{l}\right) \xi^{m},
\end{aligned}
$$

where $\mu_{k}$ denotes the unit vector with $\left(\mu_{k}\right)_{i}=\delta_{i k}$. We use the convention that $A_{j}(m), B_{j}(m)=0$ if $m$ does not belong to the basic set $M$ defined in (26). The right hand side of (20) becomes

$$
\begin{aligned}
\lambda_{j} \xi_{j} & +\lambda_{j} \sum_{m \in M} A_{j}(m) \xi^{m}+\sum_{k, l} N_{j \mid k l}\left\{\xi^{\mu_{k}+\mu_{l}}+\sum_{m \in M}\left[A_{l}\left(m-\mu_{k}\right)\right.\right. \\
& \left.\left.+A_{k}\left(m-\mu_{l}\right)\right] \xi^{m}+\sum_{m, m^{\prime} \in M} A_{k}\left(m-m^{\prime}\right) A_{l}\left(m^{\prime}\right) \xi^{m}\right\} .
\end{aligned}
$$

By equating the two sides, the linear terms cancel out. The coefficients of the second order terms $\xi^{m}$ with $|m|=2$ read

$$
\begin{gathered}
A_{j}(m)=\frac{1}{D_{j}(m)} \sum_{k, l} N_{j \mid k l} \delta\left(m, \mu_{k}+\mu_{l}\right) \quad \text { if } m \notin \mathcal{R}_{j}(\epsilon) \\
B_{j}(m)=-D_{j}(m) A_{j}(m)+\sum_{k, l} N_{j \mid k l} \delta\left(m, \mu_{k}+\mu_{l}\right) \quad \text { if } m \in \mathcal{R}_{j}(\epsilon)
\end{gathered}
$$


where $\delta\left(m, m^{\prime}\right)=1$ for $m=m^{\prime}$ and $\delta\left(m, m^{\prime}\right)=0$ for $m \neq m^{\prime}$. Note that $A_{j}(m)$ can be chosen arbitrarily if $m \in \mathcal{R}_{j}(\epsilon)$. In the case $|m|=3$ we get

$$
\begin{gathered}
A_{j}(m)=\frac{1}{D_{j}(m)}\left[2 \sum_{k, l} N_{j \mid k l} A_{k}\left(m-\mu_{l}\right)\right. \\
\left.-\sum_{k=1}^{n} \sum_{m^{\prime} \in \mathcal{R}_{k}(\epsilon)} B_{k}\left(m^{\prime}\right)\left[m_{k}-m_{k}^{\prime}+1\right] A_{j}\left(m-m^{\prime}+\mu_{k}\right)\right] \quad \text { if } m \notin \mathcal{R}_{j}(\epsilon) ; \\
B_{j}(m)=-D_{j}(m) A_{j}(m)+2 \sum_{k, l} N_{j \mid k l} A_{k}\left(m-\mu_{l}\right) \\
-\sum_{k=1}^{n} \sum_{m^{\prime} \in \mathcal{R}_{k}(\epsilon)} B_{k}\left(m^{\prime}\right)\left[m_{k}-m_{k}^{\prime}+1\right] A_{j}\left(m-m^{\prime}+\mu_{k}\right) \quad \text { if } m \in \mathcal{R}_{j}(\epsilon) .
\end{gathered}
$$

Once more $A_{j}(m)$ can be chosen arbitrarily if $m \in \mathcal{R}_{j}(\epsilon)$.

For convenience we set the resonance coefficients $A_{j}(m)=0$.

\section{Appendix B: Proof of decoupling theorem}

The forms (38) and (39) of the normalized Galerkin system imply that the dynamics of the subspace $\Xi_{\sigma}$ is independent of the higher subspaces $\Xi_{\sigma^{\prime}}$ with $\sigma^{\prime}>\sigma$ and depends only linearly on its own variables if $\sigma \geq 2$. We prove this by the method of contradiction under the conditions (36)-(37). Let us consider the system $\dot{\Xi}_{\sigma}$, and assume that i) in the normal form (31) there is a coefficient $B_{\sigma \tau}(m) \neq 0$ with $m_{\sigma^{\prime} \tau^{\prime}} \neq 0$ and $\sigma^{\prime}>\sigma$ or that ii) there exists at least one coefficient $B_{\sigma \tau}(m) \neq 0$ with $\sum_{\tau^{\prime}=1}^{E_{\sigma}} m_{\sigma \tau^{\prime}} \geq 2$ for $\sigma \geq 2$. In case i), because $|m| \geq 2$ always, and by (36) the estimates are

$$
\begin{gathered}
d_{\sigma \tau}(m) \geq\left|\Re\left(D_{\sigma \tau}(m)\right)\right| \geq\left|\rho_{\sigma^{\prime} \tau^{\prime}}+\rho_{11}-\rho_{\sigma \tau}\right|= \\
=\left|\rho_{\sigma^{\prime} \tau^{\prime}}-\rho_{\sigma \tau}\right|+\left|\rho_{11}\right| \geq \Delta_{\sigma}+\left|\rho_{11}\right|>\epsilon,
\end{gathered}
$$

which tells that $m \notin \mathcal{R}_{j}(\epsilon)$ with $j \equiv(\sigma, \tau)$ and thus $B_{\sigma \tau}(m)=0$ in contradiction to the assumption i).

In the case ii) we get at first

$$
d_{\sigma \tau}(m) \geq\left|\Re\left(D_{\sigma \tau}(m)\right)\right| \geq\left|\sum_{\sigma^{\prime}=1}^{\sigma} \sum_{\tau^{\prime}=1}^{E_{\sigma^{\prime}}} m_{\sigma^{\prime} \tau^{\prime}} \rho_{\sigma^{\prime} \tau^{\prime}}-\rho_{\sigma \tau}\right| .
$$

If now

$$
\left|\sum_{\sigma^{\prime}=1}^{\sigma} \sum_{\tau^{\prime}=1}^{E_{\sigma^{\prime}}} m_{\sigma^{\prime} \tau^{\prime}} \rho_{\sigma^{\prime} \tau^{\prime}}\right|<\left|\rho_{\sigma \tau}\right|
$$

then by diminishing the left hand side under the assumption ii) we can write

$$
2\left|\rho_{\sigma 1}\right| \leq\left|\rho_{\sigma \tau^{\prime}}\right|+\left|\rho_{\sigma \tau^{\prime \prime}}\right| \leq\left|\sum_{\sigma^{\prime}=1}^{\sigma} \sum_{\tau^{\prime}=1}^{E_{\sigma^{\prime}}} m_{\sigma^{\prime} \tau^{\prime}} \rho_{\sigma^{\prime} \tau^{\prime}}\right|<\left|\rho_{\sigma \tau}\right| \leq\left|\rho_{\sigma E_{\sigma}}\right|
$$


which implies $\left|\rho_{\sigma 1}\right|<W_{\sigma}$ and contradicts (37). In the case complementary to (77) we have

$$
d_{\sigma \tau}(m) \geq \sum_{\sigma^{\prime}=1}^{\sigma} \sum_{\tau^{\prime}=1}^{E_{\sigma^{\prime}}} m_{\sigma^{\prime} \tau^{\prime}}\left|\rho_{\sigma^{\prime} \tau^{\prime}}\right|-\left|\rho_{\sigma \tau}\right| \geq 2\left|\rho_{\sigma 1}\right|-\left|\rho_{\sigma E_{\sigma}}\right| .
$$

Making use of (37) in the form $\left|\rho_{\sigma 1}\right|>W_{\sigma}+\epsilon$ we continue (79) as

$$
d_{\sigma \tau}(m)>\left|\rho_{\sigma 1}\right|+W_{\sigma}+\epsilon-\left|\rho_{\sigma E_{\sigma}}\right|=\epsilon
$$

which means that case ii) is not contained in the resonant set $\mathcal{R}_{j}(\epsilon)$ with $j \equiv(\sigma, \tau)$. This completes the proof.

\section{Appendix C: Proof of slaving}

The dominant subspace $\Xi_{1}$ is independent of the higher subspaces $\Xi_{\sigma}, \sigma \geq 2$. Since the linear part of $\Xi_{1}$ is stable, the flow $\dot{\Xi}_{1}$ possesses a finite basin of attraction $B A$ by the theorem of Hartmann-Grobman [5]. We claim that, if the initial points $\Xi_{1}(t=0) \in B A$, then the fixed point $\xi=0$ is stable independently of the initial values $\Xi_{\sigma}(t=0)$ with $\sigma \geq 2$. We demonstrate this in an exemplary way for a three-dimensional system. The general proof merely needs more efforts in writing, but is otherwise fully analogous.

Let us assume that the first subspaces $\Xi_{1}(t), \ldots, \Xi_{\sigma-1}(t)$ are stable, and that the next subspace $\Xi_{\sigma}$ is three-dimensional with one real variable $x$ and two complex conjugate variables $r \exp (i \varphi), r \exp (-i \varphi)$ with $r \geq 0$. By (39) the corresponding dynamical system reads

$$
\begin{aligned}
& \dot{x}=\left[\lambda_{\sigma 1}+\gamma_{1}(t)\right] x+\gamma_{2}(t) r+\theta_{1}(t) ; \\
& \dot{r}=\gamma_{3}(t) x+\left[\rho_{\sigma 2}+\gamma_{4}(t)\right] r+\theta_{2}(t),
\end{aligned}
$$

where both $\rho_{\sigma 2}=\Re\left(\lambda_{\sigma 2}\right)$ and $\lambda_{\sigma 1}$ are negative, and the real coefficients $\gamma(t)$, $\theta(t)$ are determined by the variables of the lower subspaces. We need not know the phase $\varphi=\varphi(t)$. By the inductive assumption, the four functions $\gamma_{1}$ to $\gamma_{4}$ and $\theta_{1 / 2}$ become arbitrarily small for $t \rightarrow \infty$. Since the above system is linear with regular time dependent coefficients, the solutions $x(t)$ and $r(t)$ certainly

are bounded within any finite time interval. With $\lambda=\max \left\{\lambda_{\sigma 1}, \rho_{\sigma 2}\right\}<0$ the time derivative of the function $\mathcal{L}=x^{2}+r^{2}$ obeys the relation

$$
\dot{\mathcal{L}} / 2 \leq \lambda\left[x^{2}+r^{2}\right]+\gamma_{1} x^{2}+\gamma_{4} r^{2}+\left[\gamma_{2}+\gamma_{3}\right] x r+\theta_{1} x+\theta_{2} r
$$

Applying an orthogonal transformation $(x, r) \rightarrow\left(y_{1}, y_{2}\right)$ we can get rid of the mixed term $x r$. The transformed expression reads

$$
\dot{\mathcal{L}} / 2 \leq \lambda^{\prime}\left[y_{1}^{2}+y_{2}^{2}\right]+\vartheta_{1} y_{1}+\vartheta_{2} y_{2}
$$


with $\lambda^{\prime}=\lambda+\delta(t)$ where $\delta(t)$ and $\vartheta_{1 / 2}$ are arbitrarily small magnitudes if $t$ is sufficiently large. As a consequence, if we choose points with

$$
\left|y_{j}\right|>\frac{2\left|\vartheta_{j}\right|}{\left|\lambda^{\prime}\right|} ; j=1,2
$$

then $\dot{\mathcal{L}}<0$ and all initial points end asymptotically in the complementary, arbitrarily small domain $\mathcal{D}_{0}$ with $\left|y_{j}\right| \leq\left|2 \vartheta_{j} / \lambda^{\prime}\right|$. As a final step we invoke the theorem of Hartman-Grobman [5] which states that our transformed system (38), (39) is homeomorph to the linearized problem in a finite neighbourhood of the origin, provided all eigenvalues have a negative real part. If $t$ is sufficiently large, then $\mathcal{D}_{0}$ is a subset of the validity domain of the Hartman-Grobman theorem. Thus, if $t \rightarrow \infty$ then $(x, r) \rightarrow(0,0)$.

\section{Appendix D: Table of eigenvalues}

Table 2

Real and imaginary part $\Re(\lambda), \Im(\lambda)$ of the eigenvalues $\lambda_{j}$ with $j=(q, \nu)$ for $R=4320$ and $R=5000$ at $\alpha=\alpha_{c}$. The ordering is given according to the real parts at $R=4320$. The different eigenvalues at given wave number $\mathrm{q}$ are labeled by $\nu=1,2, \ldots \mathrm{S}$ and A denote symmetric and antisymmetric eigenfunctions, respectively. 


\begin{tabular}{l|r|r|c|c|c|c|c|}
\hline No. & $q$ & $\nu$ & $\mathrm{S} / \mathrm{A}$ & $\Re(\lambda)$ & $\Im(\lambda)$ & $\Re(\lambda)$ & $\Im(\lambda)$ \\
& & & & $R=4320$ & $R=4320$ & $R=5000$ & $R=5000$ \\
\hline 1 & -1 & 1 & $\mathrm{~S}$ & -0.0034297871 & 0.28323209 & -0.0015441660 & 0.27621304 \\
2 & 1 & 1 & $\mathrm{~S}$ & -0.0034297871 & -0.28323209 & -0.0015441660 & -0.27621304 \\
3 & 0 & 1 & $\mathrm{~A}$ & -0.0046737834 & 0 & -0.0040381470 & 0 \\
4 & 0 & 2 & $\mathrm{~A}$ & -0.013814702 & 0 & -0.011935903 & 0 \\
5 & 0 & 3 & $\mathrm{~A}$ & -0.027523116 & 0 & -0.023779971 & 0 \\
6 & 0 & 4 & $\mathrm{~A}$ & -0.045800419 & 0 & -0.039571561 & 0 \\
7 & -1 & 2 & $\mathrm{~S}$ & -0.053931898 & 0.96621013 & -0.050157877 & 0.97004199 \\
8 & -1 & 2 & $\mathrm{~S}$ & -0.053931898 & -0.96621013 & -0.050157877 & -0.97004199 \\
9 & 0 & 5 & $\mathrm{~A}$ & -0.068646855 & 0 & -0.059310882 & 0 \\
10 & -2 & 1 & $\mathrm{~A}$ & -0.075518479 & 1.9643698 & -0.070275192 & 1.9697702 \\
11 & 2 & 1 & $\mathrm{~A}$ & -0.075518479 & -1.9643698 & -0.070275192 & -1.9697702 \\
12 & -3 & 1 & $\mathrm{~S}$ & -0.090121159 & 2.9671465 & -0.084056136 & 2.9738632 \\
13 & 3 & 1 & $\mathrm{~S}$ & -0.090121159 & -2.9671465 & -0.084056136 & -2.9738632 \\
14 & 0 & 6 & $\mathrm{~A}$ & -0.096062496 & 0 & -0.082997997 & 0 \\
15 & 0 & 7 & $\mathrm{~A}$ & -0.12804737 & 0 & -0.11063292 & 0 \\
16 & 0 & 8 & $\mathrm{~A}$ & -0.16460149 & 0 & -0.14221568 & 0 \\
17 & 0 & 9 & $\mathrm{~A}$ & -0.20572486 & 0 & -0.17774628 & 0 \\
18 & 0 & 10 & $\mathrm{~A}$ & -0.25141749 & 0 & -0.21722471 & 0 \\
19 & 0 & 11 & $\mathrm{~A}$ & -0.30167938 & 0 & -0.26065098 & 0 \\
20 & 0 & 12 & $\mathrm{~A}$ & -0.35651052 & 0 & -0.30802509 & 0 \\
21 & 0 & 13 & $\mathrm{~A}$ & -0.41591092 & 0 & -0.35934704 & 0 \\
22 & 0 & 14 & $\mathrm{~A}$ & -0.47988059 & 0 & -0.41461683 & 0 \\
\hline
\end{tabular}

\section{Acknowledgements}

This work was supported by the grant Ra 229/2 of the Deutsche Forschungsgemeinschaft. We are thankful to Dr. Ludger Hannibal for helpful discussions and to Dr. Charilaos Kougias for giving computational advice in the beginning of this work.

\section{References}

[1] J. Carr, Application of center manifold theory, Applied mathematical science 35 (Springer, New York, 1981).

[2] L.D. Landau, On the problem of turbulence, Dokl. Akad. Nauk SSSR 44 (1944) 311-314.

[3] J.T. Stuart, On the non-linear mechanics of wave disturbances in stable and unstable parallel flows, Part 1. The basic 
behaviour in plane Poisseuille flow, J. Fluid Mech. 9 (1960) 353-370.

[4] V.I. Arnol'd, Geometrical methods in the theory of ordinary differential equations (Springer, New York, 1983).

[5] J. Guckenheimer and P. Holmes, Nonlinear oscillations, dynamical systems, and bifurcations of vector fields (Springer, New York, 1983).

[6] S. Wiggins, Introduction to applied nonlinear dynamical systems and chaos (Springer, New York, 1990).

[7] H. Haken, Synergetics (Springer, New York, 1987).

[8] A. Rauh and J. Pade, Decoupling of dissipative flows by interval normal forms in the Poincaré domain, Reports on Math. Physics 27 (1989) 179-192.

[9] J. Pade, A. Rauh and G. Tsarouhas, Application of normal forms to the Lorenz model in the subcritical region, Physica D 29 (1987) 236-246.

[10] J. Watson, On the non-linear mechanics of wave disturbances in stable and unstable parallel flows, Part 2. The development of a solution for plane Poiseuille flow, J. Fluid Mech. 9 (1960) 371-389.

[11] T. Herbert, On perturbation methods in nonlinear stability theory, J. Fluid Mech. 126 (1983) 167-186.

[12] P.K. Sen and D. Venkateswarlu, On the stability of plane Poiseuille flow to finite-amplitude disturbances, considering the higher-order Landau coefficients, J. Fluid Mech. 133 (1983) 179-206.

[13] T. Herbert, Stability of plane Poiseuille flow - theory and experiment, Fluid Dyn. Trans. 11 (1983) 77-126. 
[14] S.A. Orszag and A.T. Patera, Hydrodynamic stability of shear Flows, in Les Houches Session 36 (North-Holland, 1983) 623-662.

[15] B.J. Bayly, S.A. Orszag and T. Herbert, Instability mechanism in shear-flow transition, Ann. Rev. Fluid Mech. 20 (1988) 359-391.

[16] W. Eckhaus, Studies in non-linear stability theory (Springer, Berlin 1965).

[17] S.A. Orszag, Accurate solution of the Orr-Sommerfeld stability equation, J. Fluid Mech. 50 (1971) 689-703.

[18] L.D. Landau and E.F. Lifshitz, Fluid mechanics, Course of theoretical physics Volume 6, 2nd edition (Pergamon Press Oxford 1989).

[19] H. Schlichting, Grenzschicht-Theorie (Verlag G. Braun Karlsruhe 1982).

[20] I. Soibelman and D.I. Meiron, Finite-amplitude bifurcations in plane Poiseuille flow: two-dimensional Hopf bifurcation, J. Fluid Mech. 229 (1991) 389-416.

[21] J. Gary, R. Helgason, A matrix method for ordinary differential eigenvalue problems. J. Comp. Phys. 5 (1970) 169-187.

[22] A. Arneodo and O. Thual, Transition to chaos in a finite macroscopic system: Direct numerical simulation versus normal form prediction, in W. Güttinger and G. Dangelmayer (eds), Physics of structure formation: Theory and simulation (Springer, Berlin, 1987) 313-333.

[23] B.D. Hassard, N.D. Kazarinoff and Y.-H. Wan, Theory and application of Hopf bifurcation (Cambridge University Press, London, 1981). 


\section{Figure captions}

Fig. 1. Partitioning of the eigenvalues into groups for the decoupling theorem at $R=4320$. The eigenvalues are indicated by circles. The first stripe of width $W_{1}$ defines the dominant system; it contains the two complex conjugate eigenvalues which become critical at $R=R_{c}$ and one real eigenvalue. All other stripes containing eigenvalues have zero width here. The distances $\Delta_{\sigma}$ are all larger $\epsilon-\left|\rho_{11}\right|$, where $\epsilon$ is the interval parameter of the normal form. Note that one easily could group the eigenvalues in a different way when the distances become too small.

Fig. 2. Critical lines $\dot{r}=0$ and $\dot{\xi}=0$ in $\xi r$-space as given by the transformed dominant system (46), (47) for $R=4320$. The stable fixed point is at the origin $(0,0)$, the unstable one is denoted by $F^{*}$. The arrows indicate the direction $(\dot{r}, \dot{\xi})$ of the flow. For $r \geq 0.0427$, which is out of scale, the arrows along the curve $\dot{\xi}=0$ change sign.

Fig. 3. Critical amplitude $\eta_{c}$ (solid curve) by the normal form method as a function of the Reynolds number $R$, and stability boundaries found by direct integration of the Galerkin system (dashed curves); the up and down arrows indicate the existence of growing modes and decay of all modes, respectively. The critical amplitudes of the normal form (1) and of the Landau method (2) are compared in the insert.

Fig. 4. Dependence of the renormalized parameter $b_{L}$ on the number $N$ of the eigenfunctions with $q=0$ for $R=5770$. Curve (1) and curve (2) refer to the usual and our boundary conditions, respectively. In both cases the Orr-Sommerfeld basis functions are normalized to the same energy. 